\title{
Comparative study of the tibial tubercle- trochlear groove distance measured in two ways and tibial tubercle-posterior cruciate ligament distance in patients with patellofemoral instability
}

Lei Shu ${ }^{\dagger}$, Qubo Ni ${ }^{\dagger}$, Xu Yang, Biao Chen, Hua Wang and Liaobin Chen ${ }^{*}$

\begin{abstract}
Background: A comparative analysis of the strengths and weaknesses of three different methods for radiologic evaluation of patellofemoral instability (PFI).

Methods: Computed tomography (CT) and magnetic resonance imaging (MRI) were performed in 47 patients with or without PFI. The tibial tubercle-trochlear groove (TT-TG) distance was measured by two observers through conventional $C T$ and three-dimensional $C T$ reconstruction (TDR-TT-TG) respectively and the tibial tubercle-posterior cruciate ligament (TT-PCL) distance with MRI. The intraclass correlation coefficient (ICC) was used to evaluate the interobserver reliability. In addition, the differences of three measurements between different patients were compared. The consistency of TT-TG and TDR-TT-TG was analyzed by the Bland-Altman method.

Results: The ICCs of three measurements were high between two observers; the results were TT-TG (ICC $=0.852)$, TDR-TT-TG $(I C C=0.864)$, and TT-PCL $(I C C=0.758)$. The values of PFI patients were significantly higher than those of non-PFI patients, and the mean TT-TG, TDR-TT-TG, and TT-PCL distance in patients with PFI were $19.0 \pm 3.8 \mathrm{~mm}$, $19.0 \pm 3.7 \mathrm{~mm}$, and $25.1 \pm 3.6 \mathrm{~mm}$, respectively. There was no statistically significant difference between the TT-TG distance and the TDR-TT-TG distance, we found no significant difference. The Bland-Altman analysis showed that the TDR-TT-TG distance was in good agreement with the TT-TG distance.

Conclusion: All three methods can be used to assess PFI; the TDR-TT-TG measurement method has superior operability and better interobserver consistency. It may be an alternative method to the conventional TT-TG distance measurement.
\end{abstract}

Keywords: Patellofemoral instability, Patella dislocation, TT-TG distance, TT-TG distance under three-dimensional CT reconstruction, TT-PCL distance

\footnotetext{
* Correspondence: Ibchen@whu.edu.cn

'Lei Shu and Qubo Ni contributed equally to this study.

Department of Orthopedic Surgery, Zhongnan Hospital of Wuhan University,

Wuhan 430071, China
}

(c) The Author(s). 2020 Open Access This article is licensed under a Creative Commons Attribution 4.0 International License, which permits use, sharing, adaptation, distribution and reproduction in any medium or format, as long as you give appropriate credit to the original author(s) and the source, provide a link to the Creative Commons licence, and indicate if changes were made. The images or other third party material in this article are included in the article's Creative Commons licence, unless indicated otherwise in a credit line to the material. If material is not included in the article's Creative Commons licence and your intended use is not permitted by statutory regulation or exceeds the permitted use, you will need to obtain permission directly from the copyright holder. To view a copy of this licence, visit http://creativecommons.org/licenses/by/4.0/ The Creative Commons Public Domain Dedication waiver (http://creativecommons.org/publicdomain/zero/1.0/) applies to the data made available in this article, unless otherwise stated in a credit line to the data. 


\section{Introduction}

Patellofemoral instability (PFI) refers to the fact that the patella cannot slide normally in the femoral trochlear groove, causing one or more subluxations or dislocations and abnormal patella slippage, resulting in a series of symptoms [1]. PFI is a common disease in orthopedic sports medicine [2], especially in women aged 10-17 years [3-5]. The incidence rate is approximately between 7 and 49 people per $100,000[3,5,6]$. If the patellofemoral joint is unstable, every instance of flexion and extension of the knee joint will cause uneven stress and wear of the articular cartilage. Long-term instability can lead to serious consequences such as cartilage injury, patellofemoral joint degeneration, and quadriceps atrophy [7-9]. Although the reversed dynamic patellar apprehension test was recently introduced by Zimmermann et al. [10] as a reliable clinical examination tool for the diagnosis of PFI, but its clinical use still needs more research confirmation. At present, the diagnosis of PFI still mainly relies on the comprehensive judgment of combining the patients' medical history with a detailed physical examination and imaging procedures.

After the failure of conservative treatment for PFI, surgery is frequently needed. To date, the operation for an unstable patella is mainly based on the principle of the balancing of soft tissue and bone structure [4, 11, 12]. Bone repair procedures mainly include tibial internal osteotomy and femoral trochleoplasty [13, 14]. Whether the distal realignment procedure is performed, the distance of tibial tubercle-trochlear groove (TT-TG) is often applied [4]. This radiographic parameter was originally described by Goutallier et al. [13] in 1978 on an axial radiograph. Dejour et al. [15] first used the computed tomography (CT) technology to measure the TT-TG distance, which is considered to be an important reference for quantifying PFI. In addition, magnetic resonance imaging (MRI) has been gradually used to identify the position of the tibial tuberosity, with the advantages of evaluating soft tissue structures and the articular cartilage with no radiation [16-18]. However, the TT-TG distance measured on CT images is still considered as a gold standard [6, 19-21]. Several studies have emphasized factors that may have an effect on the value of TT-TG distance, such as trochlear dysplasia and knee rotation [16, 22, 23].To expel the influence of these variables, Seitlinger et al. [19, 23, 24] have proposed to judge PFI with MRI-the tibial tubercleposterior cruciate ligament (TT-PCL) distance. The authors believed that the TT-PCL distance is more accurate than the TT-TG distance for identifying the position of the tibial tuberosity, and that is may be an alternative modality for assessing PFI.

As we all know, the traditional measurement of TTTG requires overlapping images of two planes, and the
TT-PCL measurement requires three layers of superposition, identifying points containing the insertion point of PCL, trochlear groove, tibial tubercle, etc., whereas these markers are not in an axial plane $[25,26]$ on conventional CT or MRI, which may lead to a low intra- and interobserver reliability to measure the distances. Therefore, to reduce the measurement bias of the former techniques, we projected that PFI can be assessed by measuring the distance between the tibial tuberosity and the femoral trochlear groove with three-dimensional CT reconstruction (TDR) images. This TT-TG distance measured by use of $3 \mathrm{D}$ reconstruction is abbreviated (TDR-TT-TG). This method only requires one image to complete the measurement and has the advantage of being simple to carry out (Fig. 1c). The aim of our study was to measure the TT-TG distance by TDR in patients with or without PFI and, furthermore, to compare the measurement consistency of TT-TG distance, TDR-TTTG distance, and TT-PCL distance.

\section{Materials and methods}

Forty-seven patients with PFI were admitted to the observation group from April 2017 to April 2019 in our hospital. Selection criteria were as follows: (1) clinical diagnosis of PFI and (2) no history of knee surgery. Exclusion criteria were as follows: (1) patients with MRI or CT contraindications; (2) multiple ligament injuries, especially in patients with posterior cruciate ligament injury; (3) patients with knee deformity or severe osteoarthritis; and (4) patients with periarticular fractures. Forty-seven non-PFI patients (non-acute stage gout patients) who underwent CT and MRI examinations on the ipsilateral knee in our hospital were selected as the control group.

\section{Imaging acquisition}

CT and MRI were performed in patients with PFI, and all gout patients underwent MRI and dual-source dualenergy CT examinations. Imaging operations were completed by unified trained technicians, and related parameters used in the scanning of each patient were uniformed. The specific procedures are as follows:

\section{CT scan}

Conventional CT scan was performed on the Siemens 64-slice CT (Siemens Definition, Erlangen, Germany). All patients were supine with leg and thigh in full extension. Straps were attached on the lower leg to avoid rotation. Images were taken with the following parameters: slice thickness $4 \mathrm{~mm}$, scan time $9.28 \mathrm{~s}$ (nearly 5 to $10 \mathrm{~s}$ ), rotation time $1 \mathrm{~s}$, care dose $(120 \mathrm{kV}, 100 \mathrm{mAs})$, pitch 0.8 $\mathrm{mm}$, and matrix $512 \times 512$.

The dual-source dual-energy computed tomography (DSDECT) scan completed on the same instrument 

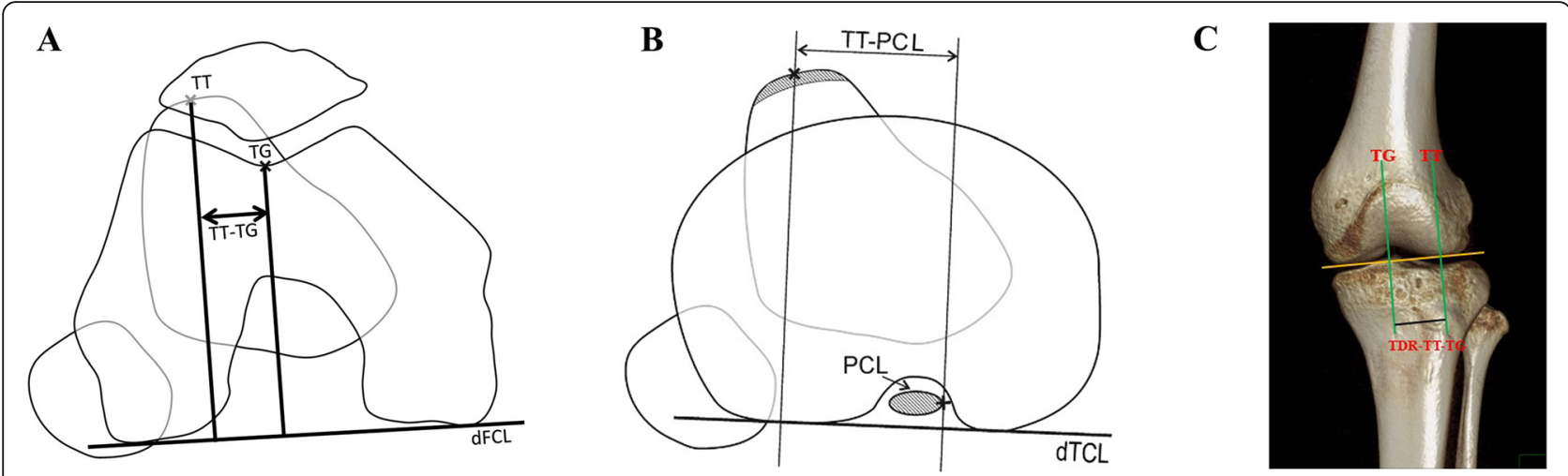

Fig. 1 Three different radiologic methods for assessment of the tibial tuberosity position in relationship to the trochlea groove and posterior cruciate ligament respectively. a TT-TG, tibial tubercle-trochlear groove distance (performed by CT). b TT-PCL, tibial tubercle-posterior cruciate ligament distance (performed by MRI). c TDR-TT-TG, TT-TG distance under three-dimensional reconstruction (performed by 3D-CT reconstruction). $\pi$, tibial tuberosity; $\mathrm{TG}$, trochlear groove; $\mathrm{dFCL}$, dorsal femoral condylar line; $\mathrm{dTCL}$, dorsal tibia condylar line

(Siemens Definition, Erlangen, Germany). The parameters were as follows: slice thickness $2 \mathrm{~mm}$, scan time $17.14 \mathrm{~s}$ (nearly 10 to $20 \mathrm{~s}$ ), rotation time $1.0 \mathrm{~s}$, care dose (tube A $140 \mathrm{kV}, 55 \mathrm{mAs}$; tube B $80 \mathrm{kV}, 55 \mathrm{mAs}$ ), pitch $0.7 \mathrm{~mm}$, and matrix $512 \times 512$.

The conventional CT image was exported with Philips IntelliSpace Portal for transforming into TDR image, the bone window was selected for reconstruction image of the knee joint, and the patella was removed by excluding the Freehand function in clip\&3D-segmentation, adjusted to the coronal position. Finally, a TDR image of the knee joint was obtained.

\section{MRI scan}

All about MRI (Philips MR Systems Ingenia $3.0 \mathrm{~T}$, Andover, Massachusetts) scanner was routine: the patients were in a supine position, with a standard knee coil center level against the lower edge of the patella. The knee and hip joint naturally extended, and the feet were braced to prevent any movement. Four MRI sequences of knee were obtained. The scanning parameters of Andover are given below: (1) coronal proton density-weighted spectral attenuated inversion recovery (PDW-SPAIR) MR images [repetition time millisecond (TR)/echo time millisecond (TE) 1940/30, field of view (FOV) $220 \mathrm{~mm} \times 179 \mathrm{~mm}$, matrix $368 \times$ 245, slice thickness $3 \mathrm{~mm}$, sections per slab 21]; (2) transverse PDW-SPAIR MR images (TR/TE 2036/30, FOV $169 \mathrm{~mm} \times 189 \mathrm{~mm}$, matrix $344 \times 264$, slice thickness $4 \mathrm{~mm}$, act slice gap $0.4 \mathrm{~mm}$, sections per slab 24); (3) sagittal T1-weighted aTSE (turbo spinecho) MR images (TR/TE 694/12, FOV $160 \mathrm{~mm} \times$ $160 \mathrm{~mm}$, matrix $308 \times 240$, slice thickness $3 \mathrm{~mm}$, act slice gap $0.3 \mathrm{~mm}$, sections per slab 24); and (4) sagittal proton density-weighted spectral inversion recovery (PDW-SPIR) MR images (TR/TE 1,554/30, FOV
$160 \times 160 \mathrm{~mm}$, matrix $292 \times 231$, slice thickness 3 $\mathrm{mm}$, act slice gap $0.3 \mathrm{~mm}$, sections per slab 24).

\section{Measurement \\ TT-TG measurement}

As previously mentioned, the TT-TG distance was only measured on CT images according to the method described by Dejour et al. [15]. Two special CT layers were chosen: the first was the level of the "Roman Arch" at the top of the femoral condyle, at the deepest point of the femoral trochlea (TG), and the second layer was the insertion center of the patellar tendon to the tibial tuberosity (TT). The TT-TG distance was calculated by superimposing two layers; TT line and TG line were perpendicular to the tangent line of the dorsal femoral condylar line (dFCL). The TT-TG distance was the distance between the two parallel lines, and the measurement accuracy was $0.1 \mathrm{~mm}$ (Fig. 1a).

\section{TT-PCL measurement}

The measurement was only performed on MRI images according to the TT-PCL distance described by Seitlinger et al. [23]. Three special scanning planes were chosen on the knee joint axial image. The first plane was the dorsal condylar line of the tibia, that was defined just below the articular surface of the tibia plateau and above the fibular head. The second plane was the medial border of the PCL, the plane was defined in the most inferior slice in which the ligament could still be clearly identified, which corresponds with the insertion of the ligament at the tibia. The third slice was the reference point of the tibial tubercle, which was located by marking the midpoint of the insertion of the patellar tendon, then the deepest image which posterior cruciate ligament $(\mathrm{PCL})$ originates on the tibial plateau was selected and marking the inner edge of the PCL, ultimately, 
choosing a slice that the patellar ligament was completely exposed and marking the center point of the patellar ligament. All planes described above were superimposed by software to obtain a new image. Two perpendicular lines were taken through the inner edge of the PCL and the midpoint of the patellar ligament. The distance between the two parallel lines was the TTPCL distance (Fig. 1b).

\section{TDR-TT-TG measurement}

The TDR-TT-TG distance was measured under TDR. First, the tangential line was taken from the two lowest points of the femoral condyle, and then draw two lines through the deepest point of the femoral trochlea (TG) and the most cephalic point of the tibial tuberosity (TT) respectively. Both TT line and TG line were perpendicular to the tangent of the distal femoral condyle. The horizontal distance between the two lines was measured, which was the TDR-TT-TG distance, with a measurement accuracy of $0.1 \mathrm{~mm}$ (Fig. 1c).

\section{Quality control}

Both observers were joint and sports doctors with more than 3 years of clinical experience and were blinded to the sex, age, the clinical diagnosis, the observation grouping, and the comparisons of individuals. All measurements were performed independently at the same time, and the intraclass correlation coefficient (ICC) was used to assess interobserver reliability to minimize the bias and confounding factors of measurement.

\section{Data extraction and analysis}

All relevant data were analyzed by SPSS 22.0 (IBM Corp., released 2013, IBM SPSS Statistics for Windows, Armonk, NY: IBM Corp). Continuous variables are expressed as the mean \pm standard deviation. The comparison between two groups was conducted with an independent-samples $T$ test and the Bland-Altman method for correlation analysis [Bland-Altman scatter plot was drawn by MedCalc v12.1.3 (MedCalc Software bvba, Ostend, Belgium)]. $P<0.05$ was considered statistically significant.

\section{Results}

\section{Basic characteristics of the included patients}

The PFI group included 19 males and 28 females, aged $11-49$ years old, with an average age of $20.7 \pm 8.0$ years; in the control group, 33 males and 14 females were included in the analysis, aged $23-56$ years old, with a mean age of $31.8 \pm 12.7$ years old.
Table 1 TT-TG versus TDR-TT-TG versus TT-PCL reliability (distance, $\mathrm{mm}, \overline{\mathrm{x}} \pm \mathrm{SD}$ )

\begin{tabular}{lllll}
\hline Parameter & Observer 1 & Observer 2 & Combined & ICC $^{\text {a }}$ \\
\hline TT-TG on CT & $19.0 \pm 4.0$ & $19.9 \pm 4.2$ & $19.5 \pm 4.1$ & 0.852 \\
TDR-TT-TG on TDR & $18.9 \pm 4.1$ & $19.1 \pm 3.9$ & $19.0 \pm 4.0$ & 0.864 \\
TT-PCL on MRI & $26.8 \pm 4.9$ & $24.8 \pm 5.2$ & $25.8 \pm 5.1$ & 0.758
\end{tabular}

$\pi$-TG tibial tubercle-trochlear groove, TDR-TT-TG tibial tubercle-trochlear groove under three-dimensional reconstruction, TDR three-dimensional CT reconstruction, $\Pi T-P C L$ tibial tubercle-posterior cruciate ligament

${ }^{\mathrm{a}}$ ICC $>0.75$ was considered to represent good agreement

\section{Interobserver differences in three measurements assessing PFI patients}

The ICCs of the three different measurement methods between the two observers are shown in Table 1. The interobserver agreements between the TT-TG measurement and the TDR-TT-TG measurement were good $(\mathrm{ICC}=0.852, \mathrm{ICC}=0.864)$, and the corresponding value of TT-PCL measurement was also good $(\mathrm{ICC}=0.758)$. The three measurements had good agreement among the different observers (ICC values > 0.75), and the TDR-TT-TG measurement has the best interobserver consistency (ICC $=0.864)$, which illustrates that the reliability of the three methods is superior.

\section{Comparison of the three methods for assessing PFI}

Compared with the control group, the values of TT-TG distance, TDR-TT-TG distance, and TT-PCL distance were obviously higher in PFI patients, and the difference was statistically significant $(P=0.000, P=0.000, P=$ 0.000 ). There was no statistically significant difference between the TT-TG distance and the TDR-TT-TG distance, we found no significant difference. The TT-PCL distance was larger than both the TT-TG distance and the TDR-TT-TG distance; meanwhile, the difference was statistically significant (Table 2).

Table 2 Comparison of three sets of data measurements ( $n=$ 47, $\mathrm{mm}$ )

\begin{tabular}{lllll}
\hline Parameter & Patient group & Control group & $t$ value & $P$ value \\
\hline TT-TG on CT & $19.0 \pm 3.8$ & $14.7 \pm 2.4$ & 6.594 & 0.000 \\
TDR-TT-TG on TDR & $19.0 \pm 3.7$ & $14.3 \pm 2.5$ & 7.226 & 0.000 \\
TT-PCL on MRI & $25.1 \pm 3.6$ & $21.5 \pm 3.4$ & 5.010 & 0.000 \\
$t$ value & -0.069 & -7.958 & -7.958 & \\
$P$ value & $0.945^{\mathrm{a}}$ & $0.000^{\mathrm{b}}$ & $0.000^{c}$ &
\end{tabular}

Statistically significant difference $(P<0.05)$

$\Pi T$-TG tibial tubercle-trochlear groove, TDR-TT-TG tibial tubercle-trochlear groove under three-dimensional reconstruction, TDR three-dimensional CT reconstruction, $\Pi T-P C L$ tibial tubercle-posterior cruciate ligament ${ }^{\mathrm{a}} \mathrm{TT}-\mathrm{TG}$ VS TDR-TT-TG

bTT-TG VS TT-PCL

${ }^{\mathrm{C}} \mathrm{TDR}-\mathrm{TT}-\mathrm{TG}$ VS TT-PCL 


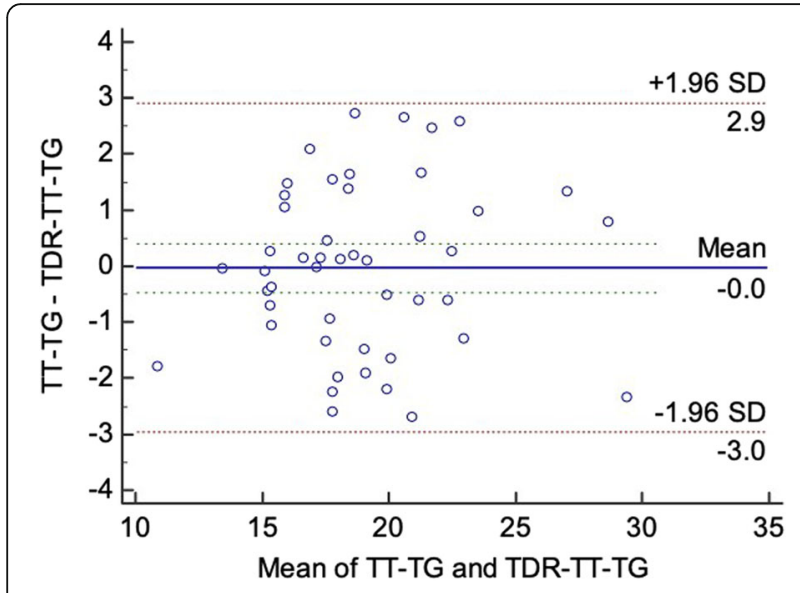

Fig. 2 Bland-Altman analysis results of the TT-TG and TDR-TT-TG distances in patients with PI. TT-TG, tibial tubercle-trochlear groove; TDR-TT-TG, tibial tubercle-trochlear groove under

three-dimensional reconstruction

\section{Bland-Altman analysis of TT-TG and TDR-TT-TG in patients with PFI}

Bland-Altman analysis of the two measurement methods showed that the mean difference between the TT-TG and TDR-TT-TG distances was $d=0.04 \mathrm{~mm}$, and the $95 \%$ limit of agreement (95\% LOA) was -2.71 to 2.78 $\mathrm{mm}$. Figure 2 demonstrates that all the points were within the LOA; the absolute value of the difference measured by the two methods was at most $2.73 \mathrm{~mm}$; moreover, the amplitude of the phase difference was clinically acceptable. Therefore, the bias of the two measurement methods was slight, and the consistency was good, indicating that the two methods can be replaced by each other.

\section{Discussion}

In this study, we used a new method based on a TDR modeling to measure TT-TG distance. The TDR-TT-TG method is only required for the completion of measurement in one layer, and our results illustrated that it has a higher interobserver consistency $(\mathrm{ICC}=0.852)$ than the other two methods in our study. Furthermore, the BlandAltman analysis indicated that the data measured by the conventional CT and TDR had good consistency, and the bias was slight; thus, the two methods are interchangeable. These results demonstrated that the TDR measurement is a reliable and feasible method. Similarly, recent research by Nha et al. [27] also illustrated that the TDR method has the advantages of smaller inter-rater variability, lower measurement bias, better reliability, and even be suitable for those patients with severe trochlear dysplasia. In their study, they established the trochlear line by connecting two deepest points of trochlear groove and the tibial tubercle line by connecting two most obvious markers, then drew a horizontal line through the midpoint of an imaginary patella to the tuberosity line as TT-TG distance. As their method requires more procedures, hence, we built the trochlear line through the deepest points of trochlear groove and the tuberosity line through the most recognizable point of tibial tuberosity. Moreover, they adjusted patients' knee in a flexion with $30^{\circ}$. However, we thought in practice that it is difficult to ensure the consistency of the patients' flexion angle and that excess flexion of knee may lead to false-negative results [6]. So we chose to have the knee fully extended for examination. Nevertheless, more well-designed studies are needed to confirm which measurement is superior. In any case, TDR images can intuitively and comprehensively display the bony structure of the knee joint, which can indicate abnormal development of the patella and femoral trochlea as well as excessive abnormalities of the tibial tuberosity.

The TT-TG distance we measured was relatively close to that of Anley et al. [19] (TT-TG $17.72 \pm 5.15 \mathrm{~mm}$ ). Additionally, in their study, the ICC of the TT-TG distance on CT was 0.97 , with a very high reliability. And their findings have also proved that the measurements for the TT-TG cannot be used interchangeably between CT and MRI, and currently accepted values for TT-TG are based on CT scans [19]. The latest review [20], which took into account the effects of different populations, confirmed that the mean TT-TG distance measured on CT for patients with PFI was $18.33 \mathrm{~mm}$, while the corresponding value was $12.85 \mathrm{~mm}$ for the control group, and they defined a new threshold of $15.5 \pm 1.5 \mathrm{~mm}$ for TT-TG distance qualified on CT, Our measurement results are different from that of this review both in patients with or without PFI. In the study of Prakash et al. [28], the mean TT-TG distance in patients with PFI was $19.05 \pm 4.8 \mathrm{~mm}$, and the value is very close to ours; meanwhile, they have assessed many factors like knee rotation angle, femoral anteversion, trochlear groove medialization, and tibial torsion. Finally, they drew a conclusion that except for tuberosity lateralization, knee rotation was an important factor affecting the TT-TG distance. In addition, the TT-TG distance may be affected by ethnicity [29]. So we speculate that these differences are related to the factors mentioned above.

We found that the mean TT-PCL distance of PFI patients was $25.1 \pm 3.6 \mathrm{~mm}$, which was larger than the TTPCL distance in the other studies [19, 30, 31]. That might be due to different MRI scanner, knee coils, and MRI protocols or due to ethnicity with for example differences in femoral and tibial rotations. As we thought, the data of three studies [19, 30, 31] above are all from Caucasians, and there was no indication of the race to which the data applied. A study of 566 children with PFI from the USA found that the average TT-PCL distance was $19.9 \mathrm{~mm}$ in normal children and $21 \mathrm{~mm}$ in children with PFI [32], and their research also found that the TT-PCL distance increased with age in the pediatric population. However, 
Boutris et al. [24] concluded that the TT-PCL threshold was identified as $21 \mathrm{~mm}$ in adults. And combined with the research results of Seitlinger et al. [23], the average TTPCL distance was $26 \pm 1.8 \mathrm{~mm}$ in adults with PFI, and they concluded the pathologic TT-PCL threshold was 24 $\mathrm{mm}$. Therefore, we believe that the determination of the TT-PCL pathological threshold needs further research.

\section{Limitations}

However, our study has some limitations. We ensured that the knee joint was in a $0^{\circ}$ flexion position during CT examination, but knee coils for MRI testing we used could result in the knee being positioned in varus with slight knee flexion. Furthermore, although we tried to keep the knee as straight as possible during the examination, the angle of knee flexion may be different due to the different sizes of the limbs. Compared to TT-PCL, the measurement of TDR-TT-TG has a certain amount of radiation in the $\mathrm{CT}$ examination and a certain subjectivity in the selection of the lowest point of the femoral trochlea and the most cephalic point of the tibial tuberosity. While the consistency between observers was good, the clinical application of the TDR-TT-TG distance requires more studies.

\section{Conclusion}

The TDR imaging technique for the measurement of the TT-TG distance has superior operability and better interobserver consistency. It may be an ideal alternative to the conventional TT-TG distance measurement.

\section{Abbreviations \\ CT: Computed tomography; DSDECT: Dual-source dual-energy computed tomography; FOV: Field of view; ICC: Intraclass correlation coefficient; LOA: Limits of agreement; MRI: Magnetic resonance imaging; PFI: Patellofemoral instability; PCL: Posterior cruciate ligament; PDW- SPAIR: Proton density-weighted spectral attenuated inversion recovery; PDW- SPIR: Proton density-weighted spectral inversion recovery; TT-TG: Tibial tubercle to trochlear groove; TT-PCL: Tibial tubercle-posterior cruciate liga- ment; TDR: Three-dimensional reconstruction; TDR-TT-TG: TT-TG distance under three-dimensional $C T$ reconstruction; TR: Repetition time millisecond; TE: Echo time millisecond; TSE: Turbo spin-echo}

\section{Acknowledgements}

The authors thank all the participants and instructors who participated in the study.

\section{Authors' contributions}

This study was developed by CLB. The first draft of the manuscript was written by SL and NQB. All authors (SL, NQB, YX, CB, WH, CLB ) contributed to the interpretation of the results and critical revision of the manuscript. All authors have approved the final manuscript before submission.

\section{Funding}

This research was financially supported by the "National Natural Science Foundation of China (81673490)" and the "National Natural Science Foundation of China (81972036)" directed by Prof. Liaobin Chen and the "National Natural Science Foundation of China(81803275) directed by Dr. Qubo Ni. All these three funding play important roles in the design, analysis, and interpretation, especially the collection of the data. They also helped in writing the manuscript, such as organization of the structure and modification of the text.

\section{Availability of data and materials}

All data are included in the manuscript.

\section{Ethics approval and consent to participate}

The Ethics Committee of Wuhan University Affiliated Zhongnan Hospital concluded that no approval is necessary for study based on its retrospective design. All patients agreed to the use of data in the study by oral consent. The oral consent approval was documented in the patients' files, which was approved by the Ethics Committee of Wuhan University Affiliated Zhongnan Hospital. All clinical investigations were conducted in accordance with the guidelines of the Declaration of Helsinki.

\section{Consent for publication}

Not applicable.

\section{Competing interests}

The authors declare no competing interests associated with this manuscript.

Received: 15 March 2020 Accepted: 25 May 2020

Published online: 08 June 2020

\section{References}

1. Magnussen RA, Verlage M, Stock E, Zurek L, Flanigan DC, Tompkins M, et al. Primary patellar dislocations without surgical stabilization or recurrence: how well are these patients really doing? Knee Surg Sports Traumatol Arthrosc. 2017;25:2352-6.

2. Haj-Mirzaian A, Thawait GK, Tanaka MJ, Demehri S. Diagnosis and characterization of patellofemoral instability: review of available imaging modalities. Sports Med Arthrosc Rev. 2017;25:64-71.

3. Gravesen KS, Kallemose T, Blønd L, Troelsen A, Barfod KW. High incidence of acute and recurrent patellar dislocations: a retrospective nationwide epidemiological study involving 24.154 primary dislocations. Knee Surg Sports Traumatol Arthrosc. 2018;26:1204-9.

4. Colvin AC, West RV. Patellar instability. J Bone Joint Surg Am. 2008;90:275162.

5. Tan SHS, Ibrahim MM, Lee ZJ, Chee YKM, Hui JH. Patellar tracking should be taken into account when measuring radiographic parameters for recurrent patellar instability. Knee Surg Sports Traumatol Arthrosc. 2018;26:3593-600.

6. Suomalainen JS, Regalado G, Joukainen A, Kääriäinen T, Könönen M, Manninen $\mathrm{H}$, et al. Effects of knee flexion and extension on the tibial tuberosity-trochlear groove (TT-TG) distance in adolescents. J Exp Orthop. 2018;5:31.

7. Sanders, T.L., Pareek, A., Johnson, N.R., Stuart, M.J., Dahm, D.L, Krych, A.J.: Patellofemoral arthritis after lateral patellar dislocation: a matched population-based analysis. Am J Sports Med 45, 1012-1017 (2017)

8. Giles LS, Webster KE, McClelland JA, Cook J. Does quadriceps atrophy exist in individuals with patellofemoral pain? A systematic literature review with meta-analysis J Orthop Sports Phys Ther. 2013:43:766-76.

9. Salonen EE, Magga T, Sillanpää PJ, Kiekara T, Mäenpää H, Mattila VM. Traumatic patellar dislocation and cartilage injury: a follow-up study of long-term cartilage deterioration. Am J Sports Med. 2017;45:1376-82.

10. Zimmermann F, Liebensteiner MC, Balcarek P. The reversed dynamic patellar apprehension test mimics anatomical complexity in lateral patellar instability. Knee Surg Sports Traumatol Arthrosc. 2019;27:604-10.

11. Longo UG, Rizzello G, Ciuffreda M, Loppini M, Baldari A, Maffulli N, et al. Elmslie-Trillat, Maquet, Fulkerson, roux Goldthwait, and other distal realignment procedures for the management of patellar dislocation: systematic review and quantitative synthesis of the literature. Arthroscopy. 2016:32:929-43.

12. Camanho GL, Bitar AC, Hernandez AJ, Olivi R. Medial patellofemoral ligament reconstruction: a novel technique using the patellar ligament. Arthroscopy. 2007;23:108.e101-4.

13. Goutallier D, Bernageau J, Lecudonnec B. The measurement of the tibial tuberosity. Patella groove distanced technique and results (author's transl). Rev Chir Orthop Reparatrice Appar Mot. 1978;64:423-8.

14. Dickschas J, Harrer J, Bayer T, Schwitulla J, Strecker W. Correlation of the tibial tuberosity-trochlear groove distance with the Q-angle. Knee Surg Sports Traumatol Arthrosc. 2016;24:915-20.

15. Dejour H, Walch G, Nove-Josserand L, Guier C. Factors of patellar instability: an anatomic radiographic study. Knee Surg Sports Traumatol Arthrosc. 1994; $2: 19-26$. 
16. Aarvold A, Pope A, Sakthivel VK, Ayer RV. MRI performed on dedicated knee coils is inaccurate for the measurement of tibial tubercle trochlear groove distance. Skelet Radiol. 2014;43:345-9.

17. Pandit S, Frampton C, Stoddart J, Lynskey T. Magnetic resonance imaging assessment of tibial tuberosity-trochlear groove distance: normal values for males and females. Int Orthop. 2011;35:1799-803.

18. Thomas S, Rupiper D, Stacy GS. Imaging of the patellofemoral joint. Clin Sports Med. 2014;33:413-36.

19. Anley CM, Morris GV, Saithna A, James SL, Snow M. Defining the role of the tibial tubercle-trochlear groove and tibial tubercle-posterior cruciate ligament distances in the work-up of patients with patellofemoral disorders. Am J Sports Med. 2015;43:1348-53.

20. Tan SHS, Lim BY, Chng KSJ, Doshi C, Wong FKL, Lim AKS, et al. The difference between computed tomography and magnetic resonance imaging measurements of tibial tubercle-trochlear groove distance for patients with or without patellofemoral instability: a systematic review and meta-analysis. J Knee Surg. 2019.

21. Hinckel BB, Gobbi RG, Kihara Filho EN, Demange MK, Pécora JR, Camanho GL. Patellar tendon-trochlear groove angle measurement: a new method for patellofemoral rotational analyses. Orthop J Sports Med. 2015;3: 2325967115601031.

22. Dietrich TJ, Betz M, Pfirrmann CWA, Koch PP, Fucentese SF. End-stage extension of the knee and its influence on tibial tuberosity-trochlear groove distance (TTG) in asymptomatic volunteers. Knee Surg Sports Traumatol Arthrosc. 2014;22:214.

23. Seitlinger G, Scheurecker G, Högler R, Labey L, Innocenti B, Hofmann S. Tibial tubercle-posterior cruciate ligament distance: a new measurement to define the position of the tibial tubercle in patients with patellar dislocation. Am J Sports Med. 2012;40:1119-25.

24. Boutris N, Delgado DA, Labis JS, McCulloch PC, Lintner DM, Harris JD. Current evidence advocates use of a new pathologic tibial tubercleposterior cruciate ligament distance threshold in patients with patellar instability. Knee Surg Sports Traumatol Arthrosc. 2018;26:2733-42.

25. Arima J, Whiteside LA, McCarthy DS, White SE, et al. J Bone Joint Surg Am. 1995;77:1331-4.

26. Whiteside, L.A., Arima, J.: The anteroposterior axis for femoral rotational alignment in valgus total knee arthroplasty. Clin Orthop Relat Res 168-172 (1995).

27. Nha K, Nam YJ, Shin MJ, Sun SD, Park JY, Debnath R, et al. Referencing the trochlear groove based on three-dimensional computed tomography imaging improves the reliability of the measurement of the tibial tuberosity-trochlear groove distance in patients with higher grades of trochlea dysplasia. Knee. 2019;26:1429-36.

28. Prakash J, Seon JK, Ahn HW, Cho KJ, Song EK. Factors affecting tibial tuberosity-trochlear groove distance in recurrent patellar dislocation. Clin Orthop Surg. 2018;10:420-6.

29. Livshetz, I., Meghpara, M.B., Karam, J.A., Salem, H.S., Mont, M.A., Hassan, O.T, Goldberg, B.A.: Do demographic or anthropometric factors affect lateralization of the tibial tubercle in a diverse population? A magnetic resonance imaging analysis. Surg Technol Int 36 (2019).

30. Daynes J, Hinckel BB, Farr J. Tibial tuberosity-posterior cruciate ligament distance. J Knee Surg. 2016;29:471-7.

31. Heidenreich MJ, Camp CL, Dahm DL, Stuart MJ, Levy BA, Krych AJ. The contribution of the tibial tubercle to patellar instability: analysis of tibial tubercle-trochlear groove (TT-TG) and tibial tubercle-posterior cruciate ligament (TT-PCL) distances. Knee Surg Sports Traumatol Arthrosc. 2017;25 2347-51.

32. Clifton B, Richter DL, Tandberg D, Ferguson M, Treme G. Evaluation of the tibial tubercle to posterior cruciate ligament distance in a pediatric patient population. J Pediatr Orthop. 2017;37:e388-93.

\section{Publisher's Note}

Springer Nature remains neutral with regard to jurisdictional claims in published maps and institutional affiliations.

\section{Ready to submit your research? Choose BMC and benefit from:}

- fast, convenient online submission

- thorough peer review by experienced researchers in your field

- rapid publication on acceptance

- support for research data, including large and complex data types

- gold Open Access which fosters wider collaboration and increased citations

- maximum visibility for your research: over $100 \mathrm{M}$ website views per year

At $\mathrm{BMC}$, research is always in progress.

Learn more biomedcentral.com/submissions 